\title{
Comparison of two commercial interferon- $\gamma$ assays for diagnosing Mycobacterium tuberculosis infection
}

\author{
J.Y. Lee*, H.J. Choi" , I-N. Park", S-B. Hong\#, Y-M. Oh\#, C-M. Lim, S.D. Lee\#, \\ Y. Koh ${ }^{\#}$, W.S. Kim ${ }^{\#}$, D.S. Kim ${ }^{\#}$, W.D. Kim ${ }^{\#}$ and T.S. Shim ${ }^{\#}$
}

ABSTRACT: The clinical usefulness of ex vivo interferon- $\gamma$ assays may largely depend on the assay format and epidemiological status of tuberculosis (TB) in the region studied.

From July 2004 to June 2005 a prospective comparison study was undertaken at a tertiary referral hospital in South Korea. The results of tuberculin skin tests (TST) and the commercially available QuantiFERON-TB Gold (QFT-G) and T SPOT-TB (SPOT) assays were compared in an intermediate TB-burden country.

Of the 224 participants studied, results from all three tests (TST, QFT-G, and SPOT) were available in 218; 87 with active TB and 131 at a low risk for TB. Using $10 \mathrm{~mm}$ as a cut-off for TST, SPOT sensitivity (96.6\%) was significantly higher than that seen for TST $(66.7 \%)$ and QFT-G (70.1\%). QFT-G showed superior specificity over TST (91.6 versus $78.6 \%$ ). Although the specificity of QFT-G was higher than that of SPOT (91.6 versus $84.7 \%$ ), the difference was not statistically significant.

Whilst some differences were found in the performance of the two commercialised interferon- $\gamma$ assays, they seemed to be superior in their detection of Mycobacterium tuberculosis infection compared with tuberculin skin tests. The most appropriate choice of interferon- $\gamma$ assay to use may depend on the clinical setting.

KEYWORDS: Interferon- $\gamma$, Mycobacterium tuberculosis, tuberculin test, tuberculosis

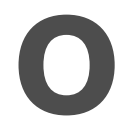
ne-third of the world's population is infected with Mycobacterium tuberculosis (MTB) [1], and tuberculosis (TB) remains a major worldwide cause of morbidity and mortality. Identifying patients with active disease and disease contacts at high risk for developing TB is particularly relevant in TB control programmes where both active disease and latent $\mathrm{TB}$ infection (LTBI) are prevalent. In South Korea, the incidence of $\mathrm{TB}$ is intermediate (92 per $100,000)$ and bacille Calmette-Guérin (BCG) vaccination is mandatory. In 2004, 65.4 per 100,000 of the general population were reported to have developed some form of $\mathrm{TB}$, and 23.9 per 100,000 of the general population developed smear-positive pulmonary TB [2]. Moreover, as the prevalence of active TB decreases and the number of TB-vulnerable subjects (e.g. elderly and immunocompromised patients) increases, physicians have become increasingly interested in chemoprophylaxis for LTBI. The first South Korean guideline for LTBI was recently introduced [3]. The target for chemoprophylaxis is

For editorial comments see page 1. complicated by the fact that the tuberculin skin test (TST) does not reliably diagnose LTBI in the BCG-vaccinated population. TST has other drawbacks, including a lack of specificity due to the purified protein derivatives (PPD) used for TST (which share some antigens with BCG and several non tuberculous mycobacteria), and low sensitivity due to false negatives among subjects who most urgently need prophylaxis for LTBI if infected with MTB, such as immunocompromised individuals $[4,5]$.

The two commercialised ex vivo interferon (IFN)$\gamma$ assays, QuantiFERON-TB Gold (QFT-G; Cellestis Limited, Carnegie, Victoria, Australia) and $\mathrm{T}$ SPOT-TB (SPOT; Oxford Immunotec, Oxford, UK), use early secretory antigenic target 6 (ESAT-6) and culture filtrate protein 10 (CFP10) as MTB-specific stimulants on ELISA and enzyme-linked immunospot assay (ELISPOT), respectively. Recently, both were approved as diagnostic tools for MTB infection [6]. Although several studies have demonstrated their high sensitivity and specificity for MTB-infected and non exposed healthy subjects [7-9], these results
AFFILIATIONS

*Division of Pulmonary Medicine and Critical Care Medicine, Dept of Internal Medicine, Konkuk University Medical Center, Chungju Hospital, Chungju-si, and

${ }^{\#}$ Division of Pulmonary and Critical Care Medicine, Dept of Internal Medicine, Asan Medical Center, College of Medicine, University of Ulsan, Seoul, South Korea.

CORRESPONDENCE

T.S. Shim

Division of Pulmonary and Critical

Care Medicine

Dept of Internal Medicine

Asan Medical Center

College of Medicine University of Ulsan

388-1 Pungnap-2dong

Songpa-gu

Seoul 138-736

South Korea

Fax: 82230106968

E-mail: shimts@amc.seoul.kr

Received:

February 042006

Accepted after revision:

April 042006

European Respiratory Journal Print ISSN 0903-1936 Online ISSN 1399-3003 
may depend on the prevalence of MTB infection, immune status, ethnicity, and so on in the population being tested [1012]. Assay-related factors might also affect IFN- $\gamma$ assay performance and interpretation. To date, no previous study has stringently compared the two commercially available ex vivo IFN- $\gamma$ assays with TST in terms of their use in the diagnosis of MTB infection in a defined population. To identify the impact of these factors, the present study directly compared the two commercial IFN- $\gamma$ assays and TST in their diagnosis of MTB infection in a defined epidemiological setting.

\section{METHODS}

\section{Subjects}

From July 2004 to June 2005, a prospective clinical study was undertaken at the Asan Medical Center (College of Medicine University of Ulsan, Seoul, South Korea), a tertiary referral medical centre at which $\sim 1,000 \mathrm{~TB}$ patients are treated annually. Due to the lack of a confirmatory test to diagnose MTB infection, it was assumed that MTB infection was present in active TB subjects and absent in subjects at low risk for TB [9]. Patients with a high clinical suspicion of active TB or for whom relevant material (sputum or biopsy specimen) was available for microbiological or pathological diagnosis were screened. Healthy, BCG-vaccinated, first or second year high school students (15-16 yrs old) with normal chest radiographs, no known history of contact with $\mathrm{TB}$ patients and no symptoms of active TB were included as a group defined as at low risk for TB.

In subgroup analysis of active $\mathrm{TB}$, "culture-confirmed $\mathrm{TB}^{\prime \prime}$ subjects were defined as culture positive for MTB; "clinical $\mathrm{TB}^{\prime \prime}$ included patients who were diagnosed histologically or who had symptoms and signs appropriate for diagnosing active TB and who responded clinically and radiologically to a full course of anti-TB treatment. "Immunocompromised" subjects were defined as having an immunosuppressive condition and included those on immunosuppressive drugs (post-transplantation, anti-cancer chemotherapy, receiving $>15 \mathrm{mg} \cdot$ day $^{-1}$ prednisone for $>1$ month), undergoing haemodialysis, who had a haematological malignancy (leukaemia, lymphoma) or diabetes mellitus $[4,9,13]$.

All participating subjects were screened using chest radiography and clinical examination, and were questioned regarding their history of exposure to $\mathrm{TB}$ patients and $\mathrm{BCG}$ vaccination. The results of serological tests for HIV were evaluated. All subjects provided written informed consent and the Institutional Review Board of the Asan Medical Center approved the study.

\section{Tuberculin skin test}

TST was performed using 2TU PPD RT23 (Statens Serum Institut, Copenhagen, Denmark) and the Mantoux method [4]. Induration size was measured using the ballpoint method after $48-72 \mathrm{~h}[14,15]$. The TST results were interpreted according to the different induration sizes, $5 \mathrm{~mm}, 10 \mathrm{~mm}$ and $15 \mathrm{~mm}$. An induration size of $10 \mathrm{~mm}$ was adopted as the cut-off value in this study. The reasons for this are as follows. 1) South Korea is still a TB-prevalent country and American Thoracic Society guidelines suggest $10 \mathrm{~mm}$ as a cut-off value in subjects from high-prevalence countries [4]. 2) Other reports published in
Asian countries also used $10 \mathrm{~mm}$ as a cut-off value for detecting MTB infection [9]. 3) South Korean guidelines for LTBI also recommended $10 \mathrm{~mm}$ as a cut-off value [3].

\section{QuantiFERON-TB Gold and T SPOT-TB}

The two IFN- $\gamma$ assays were performed according to the manufacturers' instructions [7, 9, 16, 17]. Immediately before the TST, peripheral venous blood was collected and processed within $4 \mathrm{~h}$. The samples were incubated for exactly $20 \mathrm{~h}$.

For QFT-G, the IFN- $\gamma$ value of the negative control for each subject was subtracted from the values obtained from the antigen-stimulated samples. The result was considered: "positive" if the response to the specific antigens was $\geqslant 0.35 \mathrm{IU} \cdot \mathrm{mL}^{-1}$, regardless of the value of the positive control; "negative" if the response to the specific antigens was $<0.35 \mathrm{IU} \cdot \mathrm{mL}^{-1}$ and the IFN- $\gamma$ level of the positive control was $\geqslant 0.5 \mathrm{IU} \cdot \mathrm{mL}^{-1}$; and "indeterminate" if both antigenstimulated samples were $<0.35 \mathrm{IU} \cdot \mathrm{mL}^{-1}$ and the value of the positive control was $<0.5 \mathrm{IU} \cdot \mathrm{mL}^{-1}[9]$.

For SPOT, the spot count was performed using an automated ELISPOT Reader (AID systems, Strasberg, Germany). ESAT-6or CFP-10-stimulated wells with at least five spots more than the negative control (where the negative control had fewer than or equal to five spots) were considered positive. In cases where the negative control well had six to 10 spots, the result was defined as positive when either the ESAT-6- or CFP-10stimulated well contained at least twice as many spots as the negative control well. If the negative control well had $>10$ spots, the result was considered indeterminate $[7,16,17]$.

\section{New cut-off value determination for the study population}

The optimal cut-off levels in QFT-G and SPOT were roughly calculated from a receiver operator characteristic (ROC) curve analysis using data from 87 subjects with active TB and 131 low-risk participants. For SPOT, the diagnostic criteria were simplified: the difference in spot count between antigenstimulated samples and the negative control was used as a cutoff value, irrespective of the spot count of the negative control wells. Using these new cut-off values, the sensitivities and specificities of the two IFN- $\gamma$ assays were recalculated.

\section{Statistical methods}

Differences in test performances were analysed using the Chisquared test. Concordances between the tests were assessed using the kappa coefficient. Throughout the text, confidence intervals (CI) are given as $95 \%$.

\section{RESULTS}

\section{Baseline characteristics}

The 224 screened subjects included 93 active TB patients and 131 subjects with low risk for TB. Six of the active TB subjects were excluded due to failure to measure TST induration size, leaving 218 subjects in the analysis. The mean age of the enrolled subjects was $28.6 \pm 19.0$ yrs (range 15-83 yrs) and the male to female ratio was $6.5: 1$. One hundred and seventy-six $(80.7 \%)$ subjects had received BCG vaccination (155 subjects had BCG scars) and 42 (19.3\%) had either not received BCG vaccination or did not know their BCG vaccination status. Serological HIV tests were performed in 31 active TB subjects, all with negative results. No other subjects appeared to have 


\begin{tabular}{lcc}
\hline TABLE 1 & Baseline characteristics of subjects \\
\hline Variables & Active TB & Low risk for TB \\
\hline Subjects $\mathbf{n}$ & 87 & 131 \\
Age yrs & $48.0 \pm 16.4$ & $15.7 \pm 0.5^{\#}$ \\
M:F ratio & $58: 29$ & $131: 0^{\#}$ \\
BCG vaccination & $45(51.7)$ & $131(100.0)^{\#}$ \\
TST size $\mathbf{~ m m}$ & $12.5 \pm 10.0$ & $4.0 \pm 5.4^{\#}$ \\
\hline
\end{tabular}

Data are presented as mean \pm SD or $n(\%)$, unless otherwise stated. TB: tuberculosis; M: male; F: female; BCG: bacille Calmette-Guérin; TST: tuberculin skin test. ${ }^{*}: p<0.05$ compared with active TB.

clinical indications of HIV infection. The demographical characteristics of all subjects are presented in table 1 . The active TB group consisted of 87 subjects, including 55 (63.2\%) with culture-confirmed TB and 32 (36.8\%) with clinical TB. Of these 87 patients, $81(93.1 \%)$ had pulmonary TB, three $(3.4 \%)$ had TB pleurisy and another three (3.4\%) had cervical TB lymphadenopathy. Twenty-nine (33.3\%) active TB subjects had immunocompromised conditions, specifically, diabetes mellitus $(37.9 \%)$, immunosuppressive drug use $(48.3 \%$; posttransplantation in three subjects, anti-cancer chemotherapy in nine, and prednisone use $>15 \mathrm{mg} \cdot \mathrm{day}^{-1}$ for $>1$ month in two), haematological malignancy $(10.3 \%)$ and chronic renal failure on haemodialysis $(3.4 \%)$. The 131 low-risk subjects were younger than those in the active TB group and all were male because they were selected from students (15-16 yrs of age) at a males' high school. None of the low-risk subjects were reported to have any systemic diseases affecting host immunity. The BCG vaccination rate of the low-risk subjects (100.0\%) was higher than that of the active TB subjects $(51.7 \%$; $<<0.001)$.

\section{Results of the PPD skin test and IFN- $\gamma$ assays}

The TST results based on three different cut-off values are presented in table 2. Among the 218 subjects, eight (3.7\%), all from the active TB group, showed indeterminate QFT-G results. By contrast, there were no indeterminate results in the SPOT analysis.

When $10 \mathrm{~mm}$ was used as the cut-off in TST, SPOT was more sensitive $(96.6 \%)$ than TST $(66.7 \%)$ or QFT-G $(70.1 \%$; $<0.05$ for

\begin{tabular}{|c|c|c|c|c|c|}
\hline \multirow[t]{3}{*}{ TABLE 2} & \multicolumn{5}{|c|}{$\begin{array}{l}\text { Results of the tuberculin skin test (TST) } \\
\text { according to defined cut-offs }\end{array}$} \\
\hline & & \multirow{2}{*}{$\begin{array}{c}\text { Subjects } \\
\mathbf{n}\end{array}$} & \multicolumn{3}{|c|}{ TST+ } \\
\hline & & & $\geqslant 5 \mathrm{~mm}$ & $\geqslant 10 \mathrm{~mm}$ & $\geqslant 15 \mathrm{~mm}$ \\
\hline \multicolumn{2}{|c|}{ Active TB } & 87 & $64(73.6)$ & $58(66.7)$ & $38(43.7)$ \\
\hline \multicolumn{2}{|c|}{ Immunocompetent } & 58 & $50(86.2)$ & $48(82.8)$ & $30(51.7)$ \\
\hline \multicolumn{2}{|c|}{ Immunocompromised } & 29 & $14(48.3)$ & $10(34.5)$ & $8(27.6)$ \\
\hline \multicolumn{2}{|c|}{ Culture positive } & 55 & $44(80.0)$ & $39(70.5)$ & $26(47.3)$ \\
\hline \multicolumn{2}{|c|}{ Low risk for TB } & 131 & $44(33.6)$ & $28(21.4)$ & $6(4.6)$ \\
\hline
\end{tabular}

each; table 3) and QFT-G showed superior specificity over TST ( 91.6 versus $78.6 \% ; p=0.005)$. Although the specificity of QFT-G was higher than that of SPOT (91.6 versus $84.7 \%$ ), the difference was not statistically significant $(\mathrm{p}=0.13$; table 3$)$. There were no significant differences in the sensitivity of the three tests between culture-confirmed and clinical TB subjects and between BCG-vaccinated and unvaccinated subjects (data not shown). In immunocompromised subjects, the sensitivity of SPOT $(100.0 \%)$ was significantly higher than that of TST $(34.5 \%)$ or QFT-G (62.1\%), and the sensitivity of QFT-G showed a tendency to be higher compared with TST $(p=0.065)$.

Even though $5 \mathrm{~mm}$ was used as a cut-off in TST, SPOT was still more sensitive $(96.6 \%)$ than TST $(73.6 \%)$ and the sensitivities of TST and QFT-G (70.1\%) were comparable to each other. When $15 \mathrm{~mm}$ was used as a cut-off in TST, the specificities of TST (95.4\%) and QFT-G (91.6\%) were comparable to each other even though the specificity of TST was higher than that of SPOT $(84.7 \%)$. However, when $15 \mathrm{~mm}$ was used as a cut-off in TST, TST showed too low a level of sensitivity (43.7\%).

\section{Discordance among the test results}

After excluding the eight subjects with indeterminate QFT-G results, there was good overall agreement among the test results. The kappa values between TST and QFT-G, TST and SPOT, and QFT-G and SPOT were 73.3\% (kappa =0.431), 75.2\% $($ kappa $=0.496)$, and $79.0 \%($ kappa $=0.569)$, respectively.

Among the 79 active TB subjects, 17 (21.5\%) had discordant results between QFT-G and SPOT. Most (94.2\%) were QFT-G-/ SPOT+ and only one (5.8\%; culture-confirmed TB) was QFTG+/SPOT-, suggesting that SPOT was more sensitive than QFT-G for diagnosing MTB infection (fig. 1). Among the 60 QFT-G+/SPOT+ subjects, $14(23.3 \%)$ were TST-negative, and many of them $(64.3 \%)$ were immunocompromised subjects, suggesting that IFN- $\gamma$ assays are more sensitive than TST in diagnosing MTB infection, especially in immunocompromised subjects.

In 131 low-risk subjects, two (1.5\%) were TST+/QFT-G+/ SPOT+, suggesting that they might in fact be infected with MTB or another ESAT-6- or CFP-10-containing non tuberculous mycobacteria (fig. 2). Among the 102 subjects with QFTG-/SPOT- results, 17 showed TST+ results, suggesting that $\mathrm{TST}+$ results might be induced by previous BCG vaccination. Of the 131 subjects, $27(20.6 \%)$ had discordant results between QFT-G and SPOT: nine were QFT-G+/SPOT- and 18 were QFT-G-/SPOT+. Eight of the nine QFT-G+/SPOT- subjects were also TST-, suggesting that the QFT-G results might be false positives. The 18 QFT-G-/SPOT+ subjects consisted of eight $(44.4 \%)$ TST+ and $10(55.6 \%)$ TST-. The reason for this discrepancy is unclear; possible explanations would be that the SPOT results were false positives or that the subjects were truly infected with MTB or another ESAT-6- or CFP-10-containing non tuberculous mycobacteria.

\section{Best cut-off value determination}

The above analyses were performed using the manufacturerrecommended cut-off values (fig. 3). To examine whether the application of population-specific cut-off values could improve these analyses, ROC curve analysis was used to determine the best cut-off values that yielded both higher sensitivity and 


\begin{tabular}{|c|c|c|}
\hline \multirow[t]{2}{*}{ TABLE 3} & \multicolumn{2}{|c|}{$\begin{array}{l}\text { Comparison of the two interferon- } \gamma \text { assays and } \\
\text { tuberculin skin test (TST) results in each group }\end{array}$} \\
\hline & Active TB & Low risk for TB \\
\hline Subjects n & 87 & 131 \\
\hline TST $+\geqslant 10 \mathrm{~mm}$ & $58(66.7)$ & $28(21.4)$ \\
\hline QFT-G+ & $61(70.1)$ & $11(8.4)^{\#}$ \\
\hline SPOT+ & $83(96.6)^{\varphi,+}$ & 20 (15.3) \\
\hline \multicolumn{3}{|c|}{ 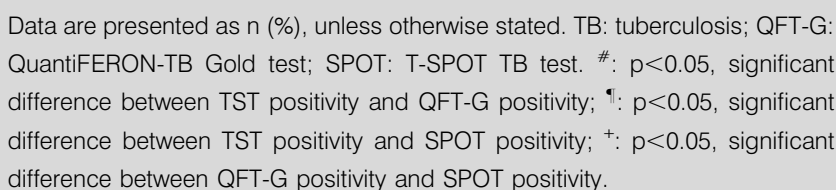 } \\
\hline
\end{tabular}

specificity (fig. 4). For QFT-G, a cut-off value of $>0.13 \mathrm{IU} \cdot \mathrm{mL}^{-1}$ of IFN- $\gamma$ level improved sensitivity $(86.2 \%$; 95\% CI 77.1-92.7), with a minor decrease in specificity $(87.0 \%$; 95\% CI 80.0-92.2). For SPOT, a cut-off value of more than seven spot counts improved specificity $(91.6 \%$; 95\% CI 85.5-95.7), with a minor decrease in sensitivity $(92.0 \%$; $95 \%$ CI $84.1-96.7)$. When the cutoff levels calculated from a ROC analysis were adopted in this study population, the sensitivities of SPOT (92.0\%) and QFT-G $(86.2 \%)$ were comparable to each other and were higher than that of TST (66.7\%). The specificities of SPOT (91.6\%) and QFT-G $(87.0 \%)$ were comparable to each other and the specificity of SPOT was higher than that of TST $(78.6 \% ; \mathrm{p}=0.005)$.

\section{DISCUSSION}

The present study reports the results of the first ever comparison of two commercial ex vivo ELISA- and ELISPOTbased IFN- $\gamma$ assays with TST for the diagnosis of MTB infection in an intermediate-burden country. Although previous studies have compared ELISA- and ELISPOT-based IFN- $\gamma$ assays [18, 19], no other work has stringently compared the two commercially available assays considered here. In the present study, when the manufacturer-recommended cut-offs were adopted, the sensitivity of SPOT was significantly higher than those of TST or QFT-G, especially in immunocompromised subjects, and the specificity of QFT-G was significantly higher than that of TST.

The two commercialised IFN- $\gamma$ assays are similar in terms of the antigens used (ESAT-6 and CFP-10) and the incubation time (overnight or 16-24 h). The main differences between the two assays lie in the technique of IFN- $\gamma$ detection (ELISA versus ELISPOT) and the specimens used (whole blood versus mononuclear cells). Of the two assays, QFT-G seems to be more convenient than SPOT, as it tests whole blood instead of mononuclear cells and because ELISA is more commonly used and simpler to perform than ELISPOT. In addition, the ELISPOT assay requires an expensive ELISPOT reader for accuracy. However, the ELISPOT assay is sensitive enough to detect single IFN- $\gamma$-producing cells.

The main theoretical advantage of IFN- $\gamma$ assays using MTBspecific antigens is a high specificity in the diagnosis of MTB infection [9, 20-22]. However, there is controversy regarding the degree to which the sensitivity of IFN- $\gamma$ assays exceeds that

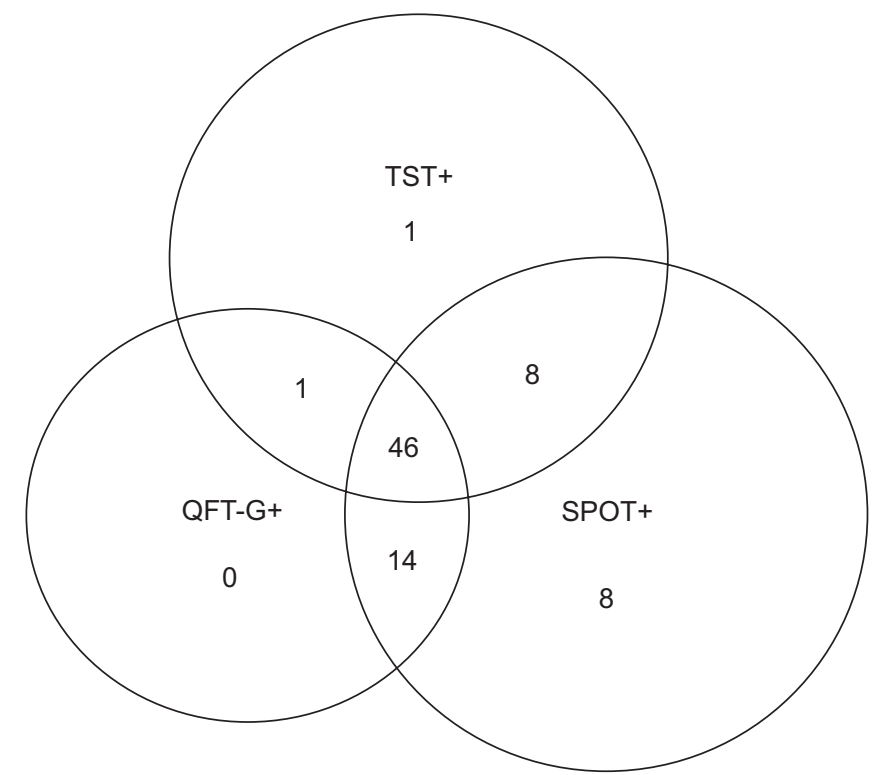

FIGURE 1. Venn diagram representing the number of positive results from tuberculin skin tests (TST), QuantiFERON-TB Gold tests (QFT-G) and T-SPOT-TB tests (SPOT) among the active tuberculosis subjects. TST, QFT-G, and SPOT were all negative in one subject.

of TST. In this study, when the manufacturer-recommended cut-off values were adopted, QFT-G was superior in terms of specificity $(91.6 \%)$ and SPOT was superior in terms of sensitivity $(96.6 \%)$. However, when new, population-specific cut-off values $\left(>0.13 \mathrm{IU} \cdot \mathrm{mL}^{-1}\right.$ in QFT-G and more than seven spot counts in SPOT) were adopted, the sensitivity of QFT-G

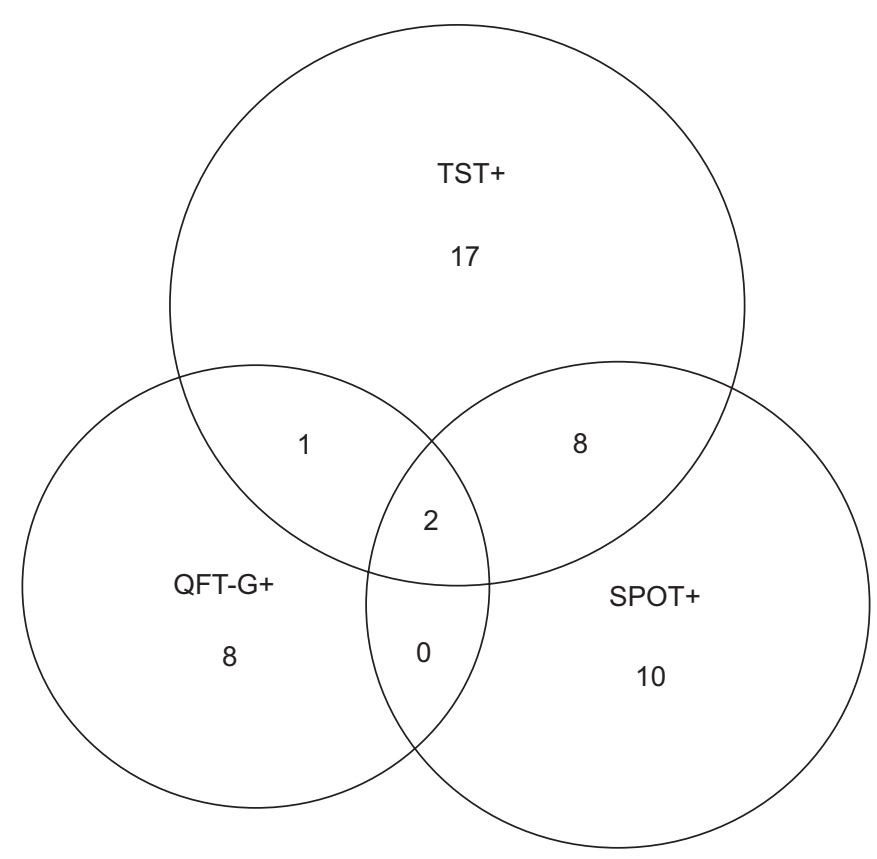

FIGURE 2. Venn diagram representing the number of positive results from tuberculin skin tests (TST), QuantiFERON-TB Gold tests (QFT-G) and T-SPOT-TB tests (SPOT) among the subjects at low risk for tuberculosis. TST, QFT-G, and SPOT were all negative in 85 subjects. 

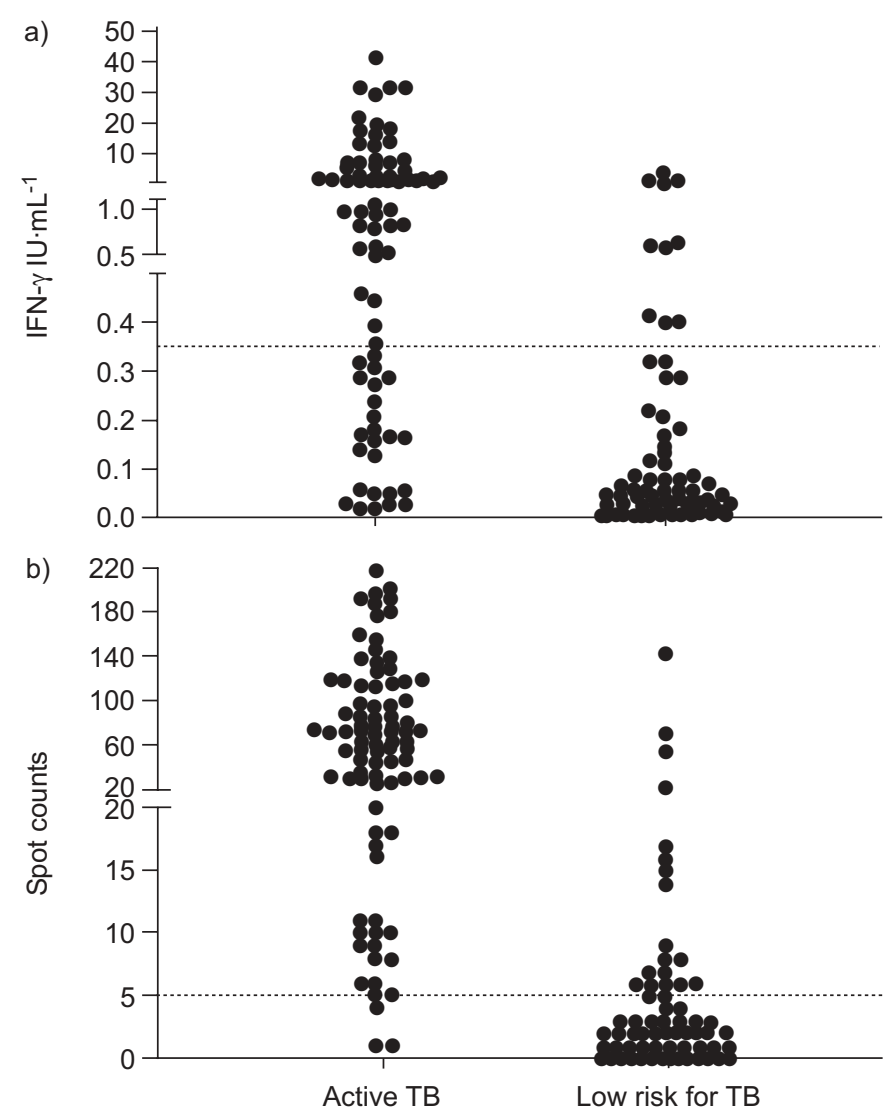

FIGURE 3. Dot plots of QuantiFERON-TB Gold and T SPOT-TB analysis. a) Interferon (IFN)- $\gamma$ level and b) number of IFN- $\gamma$-producing cells responding to early secretory antigenic target 6 or culture filtrate protein 10 in active tuberculosis (TB) subjects ( $n=87)$ and subjects at low risk for TB $(n=131)$. .......... cut-off value recommended by the manufacturer. Each dot represents an individual subject.

rose to $86.2 \%$ with minimal loss of specificity (91.6 to $87.0 \%$ ), and the specificity of SPOT rose to $91.6 \%$ with minimal loss of sensitivity (96.6 to $92.0 \%$ ). As a result, the QFT-G and SPOT assays showed similar sensitivities and specificities. In addition, the overall agreement between QFT-G and SPOT improved from $79.0 \%(\mathrm{kappa}=0.569)$ to $83.8 \%(\mathrm{kappa}=0.665)$. Recently, HARADA et al. [23] also suggested that the cut-off value of QFT-G should be reset at a lower level when the test is applied to a "high prevalence" situation. These findings suggest that $e x$ vivo IFN- $\gamma$ assays are superior in the diagnosis of MTB infection compared with TST. However, the performance of IFN- $\gamma$ assays may depend on epidemiological status, ethnicity, underlying diseases, and so on. Such new cut-off values should therefore be validated prospectively before being used in clinical practice. Further studies are needed to compare the usefulness of the two commercialised IFN- $\gamma$ assays in clinical practice based on their applicability (equipment, labour time, etc.) as well as on the performance of the tests.

Comparison of the data obtained from the IFN- $\gamma$ assays and TST is inherently limited by the lack of a gold standard method for diagnosing MTB infection. Even though the cause of discrepant QFT-G-/SPOT+ results in active TB subjects seems to be due to the different sensitivities of the two tests, it is not
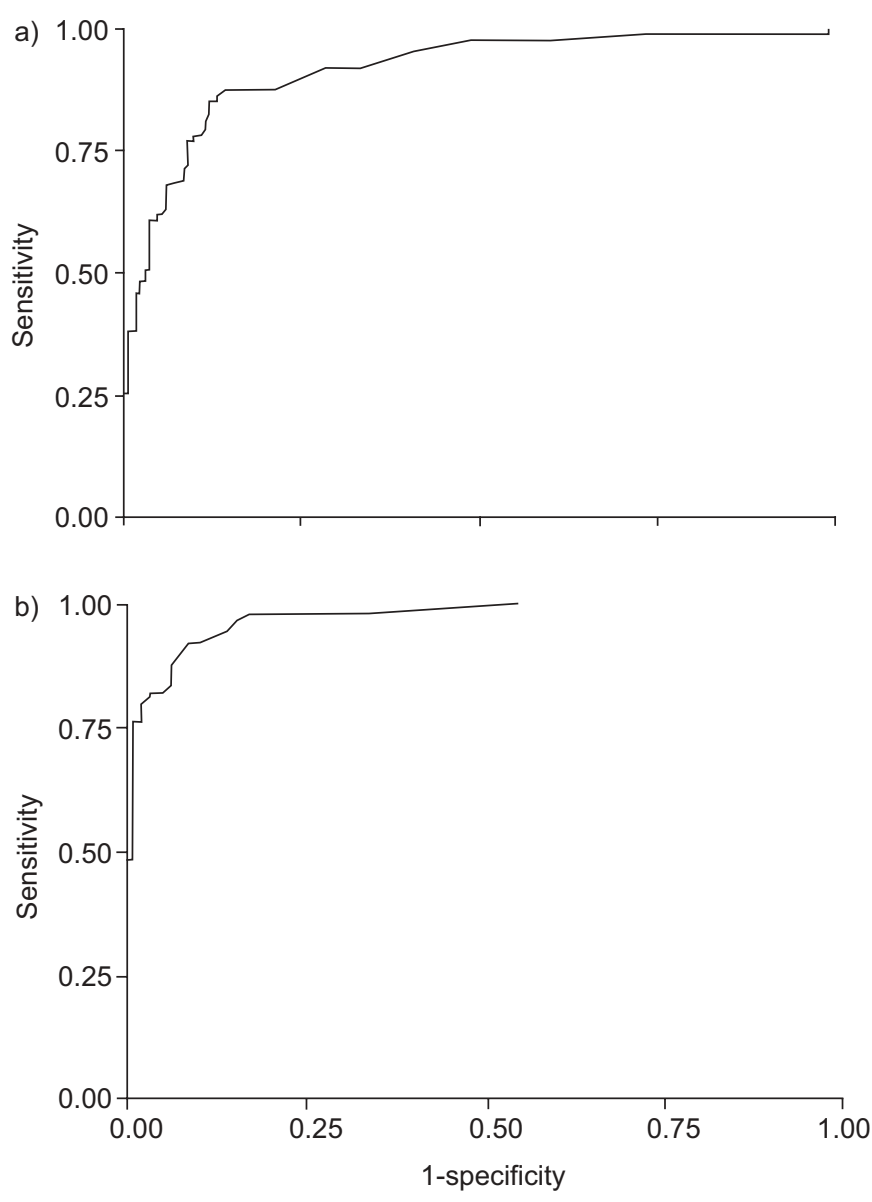

FIGURE 4. Receiver operator characteristic curves. a) Interferon (IFN)- $\gamma$ level $\left(\mathrm{IU} \cdot \mathrm{mL}^{-1}\right)$ and b) number of IFN- $\gamma$-producing cells responding to early secretory antigenic target 6 or culture filtrate protein 10 in active tuberculosis (TB) subjects $(n=87)$ and subjects at low risk for TB $(n=131)$. a) Sensitivity $86.2 \%$, specificity $87.0 \%$ and criteria IFN- $\gamma>0.13 \mathrm{IU} \cdot \mathrm{mL}^{-1}$; b) sensitivity $92.0 \%$, specificity $91.6 \%$ and criteria spot-forming cells $>7$.

possible to clearly explain the reason for the discrepant results between both IFN- $\gamma$ assays in the low-risk subjects. These issues can only be resolved by a long-term follow-up study.

One disadvantage of IFN- $\gamma$ assays is that they can yield indeterminate results. FERRARA et al. [24] found that indeterminate results were common (21\%) when using QFT-G assay. However, the majority of them were TST- or immunosuppressed [24]. In the present study, only eight (3.7\%) out of 218 subjects had indeterminate QFT-G results, and five of them were immunocompromised. In contrast to QFT-G, no indeterminate results were obtained from the SPOT assay in this study.

IFN- $\gamma$ assays have the added disadvantages of higher costs and laboratory requirements compared with TST. These higher costs should, however, be offset by savings in the long term derived from avoiding unnecessary chemoprophylaxis and reducing the number of cases of active TB. This issue is not addressed in the current study. Further studies focusing on the costs and applicability of the commercialised IFN- $\gamma$ assays are required. 
The present study has several limitations. First, some of the low-risk subjects may actually have been infected with MTB and this might lead to an underestimation of the specificity of the IFN- $\gamma$ assays. According to a South Korean national survey conducted in 1995, the MTB infection rate was $\sim 15 \%$ in $15 \mathrm{yr}$ olds but rose dramatically to about $52-60 \%$ in $18-33$ yr olds [25]. Accordingly, 15-16-yr-old students were selected as a low-risk group because they have a lower chance of being infected with MTB. As a result, only two out of 131 subjects showed positive results for all three tests.

A second possible limitation of the present study was that while a positive culture of MTB is standard for the diagnosis of active TB, some cases of active TB were diagnosed based on clinical grounds rather than bacteriological confirmation. This might lead to an underestimation of the sensitivities of the IFN- $\gamma$ assays. However, even when the analysis was restricted to culture-confirmed TB patients, there were no significant differences in the comparison results among the three tests.

In conclusion, whilst there were some differences in the performance of the two commercialised interferon- $\gamma$ assays (high sensitivity of T-SPOT-TB and probable high specificity of QuantiFERON-TB Gold), they seemed to be superior in detecting Mycobacterium tuberculosis infection compared with tuberculin skin test. The most appropriate test to use may depend on the clinical setting. As with the tuberculin skin test, the results of each test should be interpreted with caution, taking into consideration the epidemiological status of tuberculosis in the region studied and individual immunological conditions, which might decrease the responsiveness of each assay.

\section{ACKNOWLEDGEMENTS}

The authors would like to thank Woongbe Meditech (Seoul, South Korea) for their QuantiFERON-TB Gold assay kits and ResearchNET Inc. (Seoul, South Korea) for their donations and technical support. Finally, the authors also extend their thanks to the volunteers who agreed to participate in the study.

\section{REFERENCES}

1 Arend SM, Engelhard AC, Groot G, et al. Tuberculin skin testing compared with T-cell responses to Mycobacterium tuberculosis-specific and nonspecific antigens for detection of latent infection in persons with recent tuberculosis contact. Clin Diagn Lab Immunol 2001; 8: 1089-1096.

2 Annual report on the notified tuberculosis patients in Korea. Seoul: Korean Center for Disease Control and Prevention, Korean Institute for Tuberculosis, 2005.

3 Shim TS, Koh WJ, Yim JJ, Lew WJ. Diagnosis and treatment of latent tuberculosis infection in Korea. Tuberc Respir Dis 2004; 57: 101-117.

4 Geiter LJ, Gordin FM, Hershfield E, et al. Targeted tuberculin testing and treatment of latent tuberculosis infection. Am J Respir Crit Care Med 2000; 161: S221-S247.

5 Huebner RE, Schein MF, Bass JB Jr. The tuberculin skin test. Clin Infect Dis 1993; 17: 968-975.

6 Mazurek GH, Jereb J, LoBue P, Iademarco MF, Metchock B, Vernon A. Guidelines for using the QuantiFERON-TB Gold test for detecting Mycobacterium tuberculosis infection, United States. MMWR Recomm Rep 2005; 54: 49-55.
7 Lalvani A, Pathan AA, McShane H, et al. Rapid detection of Mycobacterium tuberculosis infection by enumeration of antigen-specific T cells. Am J Respir Crit Care Med 2001; 163: 824-828.

8 Fietta A, Meloni F, Cascina A, et al. Comparison of a wholeblood interferon-gamma assay and tuberculin skin testing in patients with active tuberculosis and individuals at high or low risk of Mycobacterium tuberculosis infection. Am J Infect Control 2003; 31: 347-353.

9 Mori T, Sakatani M, Yamagishi F, et al. Specific detection of tuberculosis infection: an interferon-gamma based assay using new antigens. Am J Respir Crit Care Med 2004; 170: 59-64.

10 Lalvani A, Nagvenkar P, Udwadia Z, et al. Enumeration of $\mathrm{T}$ cells specific for RD1-encoded antigens suggests a high prevalence of latent Mycobacterium tuberculosis infection in healthy urban Indians. J Infect Dis 2001; 183: 469-477.

11 Richeldi L, Ewer K, Losi M, et al. T cell-based tracking of multidrug resistant tuberculosis infection after brief exposure. Am J Respir Crit Care Med 2004; 170: 288-295.

12 Ewer K, Deeks J, Alvarez L, et al. Comparison of T-cellbased assay with tuberculin skin test for diagnosis of Mycobacterium tuberculosis infection in a school tuberculosis outbreak. Lancet 2003; 361: 1168-1173.

13 Pai M, Riley LW, Colford JM Jr. Interferon-gamma assays in the immunodiagnosis of tuberculosis: a systematic review. Lancet Infect Dis 2004; 4: 761-776.

14 Sokal JE. Measurement of delayed skin-test responses. $N$ Engl J Med 1975; 293: 501-502.

15 Dunlap NE, Bass J, Fujiwara P, et al. Diagnostic standards and classification of tuberculosis in adults and children. Am J Respir Crit Care Med 2000; 161: 1376-1395.

16 Lalvani A, Brookes R, Hambleton S, Britton WJ, Hill AV, McMichael AJ. Rapid effector function in CD8+ memory T cells. J Exp Med 1997; 186: 859-865.

17 Lalvani A. Spotting latent infection: the path to better tuberculosis control. Thorax 2003; 58: 916-918.

18 Goletti D, Vincenti D, Carrara S, et al. Selected RD1 peptides for active tuberculosis diagnosis: comparison of a gamma interferon whole-blood enzyme-linked immunosorbent assay and an enzyme-linked immunospot assay. Clin Diagn Lab Immunol 2005; 12: 1311-1316.

19 Scholvinck E, Wilkinson KA, Whelan AO, Martineau AR, Levin M, Wilkinson RJ. Gamma interferon-based immunodiagnosis of tuberculosis: comparison between wholeblood and enzyme-linked immunospot methods. J Clin Microbiol 2004; 42: 829-831.

20 Ravn P, Munk ME, Andersen AB, et al. Reactivation of tuberculosis during immunosuppressive treatment in a patient with a positive QuantiFERON-RD1 test. Scand J Infect Dis 2004; 36: 499-501.

21 Brock I, Weldingh K, Lillebaek T, Follmann F, Andersen P. Comparison of tuberculin skin test and new specific blood test in tuberculosis contacts. Am J Respir Crit Care Med 2004; 170: 65-69.

22 Chapman AL, Munkanta M, Wilkinson KA, et al. Rapid detection of active and latent tuberculosis infection in HIVpositive individuals by enumeration of Mycobacterium tuberculosis-specific T cells. AIDS 2002; 16: 2285-2293.

23 Harada N, Higuchi K, Sekiya Y, Rothel J, Kitoh T, Mori T. Basic characteristics of a novel diagnostic method 
(QuantiFERON TB-2G) for latent tuberculosis infection with the use of Mycobacterium tuberculosis-specific antigens, ESAT-6 and CFP-10. Kekkaku 2004; 79: 725-735.

24 Ferrara G, Losi M, Meacci M, et al. Routine hospital use of a commercial whole blood interferon-gamma assay for tuberculosis infection. Am J Respir Crit Care Med 2005; 172: 631-635.

25 Hong YP, Kim SJ, Lew NJ, Lee EN, Han YC. The seventh nationwide tuberculosis prevalence survey in Korea, 1995. Int J Tuberc Lung Dis 1998; 2: 27-36. 